\title{
Epigenome reprogramming - of mice and men
}

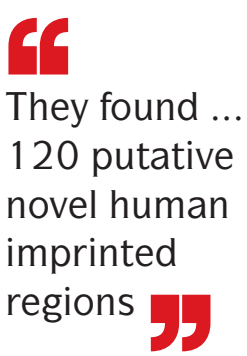

Two new studies have characterized the genome-scale landscapes of DNA methylation in human early embryos. Although the broad principles of how the epigenome is reprogrammed during early embryonic development seem to be conserved from mice to humans, various human-specific features may have interesting biological implications.

Guo et al. and Smith et al. obtained various samples from human donors that represented different stages of human fertilization and embryonic development, from gametes through to embryonic and fetal samples. The teams used reduced representation bisulphite sequencing (RRBS), in which a DNA-methylation-sensitive restriction enzyme facilitates the enrichment of methylated DNA from scarce input samples, followed by bisulphite treatment and high-throughput sequencing to characterize cytosine methylation at single-nucleotide resolution.

Both groups found that fertilization results in rapid and global demethylation of the maternal and paternal genomes to clear epigenetic memory from the previous generation and to set up the pluripotent state. This hypomethylation persists through the pre-uterine-implantation embryonic stages but is followed by widespread remethylation in post-implantation cells. These events broadly mirror those in mice, although Guo et al. used a time-course series of preimplantation embryos across their earliest cell divisions to show that the demethylation was slightly slower in humans than in mice, as it was largely complete by the twocell stage rather than the one-cell (zygotic) stage. This result might mean that similar reprogramming events during somatic cell nuclear transfer could require more time in human cells than in mouse cells.

Beyond global patterns of DNA methylation changes, both groups characterized particular loci of interest. They searched for imprinted loci, for which one of the two parental alleles resists the zygotic global demethylation, resulting in differentially methylated regions (DMRs). Smith et al. found that maternally hypermethylated DMRs were highly divergent between humans and mice during the pre-implantation stage but that most of these DMRs were transient, as they were no longer differentially methylated in embryonic stem cells derived in vitro (which were used as a proxy for unavailable early-stage post-implantation embryos) or in later post-implantation fetal samples. However, Guo et al. characterized their post-implantation tissue (6-10 weeks gestation) additionally through whole-genome bisulphite sequencing (WGBS) for a more comprehensive analysis than RRBS. They found numerous persistent
DMRs, including known imprinted regions and 120 putative novel human imprinted regions.

Finally, both teams analysed families of transposable elements (TEs), which are generally silenced by promoter DNA methylation in differentiated cell types to minimize the threat to genome stability from TE mobilization. Combining DNA methylation and transcriptomic data, they found that various TEs become demethylated and expressed during the period of global hypomethylation in pre-implantation embryonic cells. The precise timings and degrees of derepression were dependent on the specific family of TEs, and this variability in expression was greater than that for mouse TEs. Importantly, Guo et al. showed that for various TE families, evolutionarily younger TEs retain greater levels of DNA methylation, probably because these TEs have acquired fewer deleterious mutations than older TEs and thus pose a greater mobilization risk that cells must actively control. Additionally, Smith et al. found that a particularly young subfamily of the primate-specific L1PA TEs seems to have evaded this repressive mechanism through a $\sim 130$-bp deletion relative to ancestral sequences, which may explain the active transposition of these particular elements in humans, including in human cancers.

Overall, these findings provide some reassurance that studies of epigenome reprogramming in mice seem to be broadly applicable to the equivalent processes in humans; however, future characterization of the human-specific features may provide interesting insights into human biology.

Darren J. Burgess

ORIGINAL RESEARCH PAPERS Guo, H. et al.

The DNA methylation landscape of human early embryos. Nature 511, 606-610 (2014) | Smith, Z. D. et al. DNA methylation dynamics of the human preimplantation embryo. Nature $\mathbf{5 1 1}$ 611-615 (2014) 